\title{
Recubrimientos antirreflectantes de sílice sobre vidrio producidos por sol-gel
}

\author{
A. MORALES ${ }^{1}$, M.C. BAUTISTA ${ }^{1}$, D. CÁCERES ${ }^{2}$ \\ ${ }^{1}$ CIEMAT, Departamento de Energías Renovables \\ ${ }^{2}$ Departamento de Física. Escuela Politécnica Superior. Universidad Carlos III
}

\begin{abstract}
Hasta el momento no se ha preparado ningún recubrimiento antirreflectante de sílice sobre vidrio con elevadas prestaciones ópticas y buenas propiedades mecánicas que permitan la limpieza de la cubierta del colector con agua a presión sin dañar la película antirreflectante. En este trabajo se muestra un recubrimientos poroso de sílice, preparado mediante la técnica sol-gel, siguiendo la ruta polimérica con el uso de un aditivo promotor de la porosidad.

La transmitancia solar del vidrio aumenta de 0,915 a 0,970 cuando es recubierto por ambas caras con el recubrimiento antirreflectante, lo que permite un aumento considerable del rendimiento en colectores solares. Las propiedades mecánicas del recubrimiento son buenas, permitiendo la limpieza de la cubierta del colector sin daños en la película antirreflectante.

Palabras clave: sol-gel, silice, recubrimiento antirreflectante, propiedades ópticas

Anti-reflecting silica coatings on glass produced by sol-gel.

Up to now antireflective silica thin films deposited on glass have not enough strength and poor adhesion to the substrate that do not allow glass cover cleaning. Polymeric route has been used to obtain silica antireflective films on glass with the addition of an additive that introduces a certain degree of porosity in the silica films. Porous silica layers, obtained from the polymeric route, permit a considerable reduction of these light reflections compared with uncoated glasses in all the studied cases.

Solar transmittance is increased from 0.915 for the bare slide up to 0.970 for the silica thickness optimised coated sample.
\end{abstract}

Key words: sol-gel, silica thin films, antireflective coatings, optical properties

\section{INTRODUCCIÓN}

Los colectores solares térmicos presentan, independientemente del tipo de aplicación y temperatura de operación, una cubierta de vidrio para reducir las pérdidas térmicas por conducción-convección. Generalmente se emplea vidrio con bajo contenido en hierro en colectores planos y vidrio borosilicato en cilindro-parabólicos. Estos vidrios presentan, para los espesores usuales, una transmitancia entre $0,90 \mathrm{y}$ 0,92 , de forma que en el vidrio se pierde entre un 8 y $10 \%$ de la radiación solar disponible. Con el fin de reducir estas pérdidas se emplean recubrimientos antirreflectantes $(1,2)$.

El mejor material antirreflectante para el vidrio es la sílice que presenta un índice de refracción muy bajo (1.45), frente a 1,52 que presenta el vidrio, lo que permite sólo una ligera reducción de las pérdidas por reflexión. Con el fin de reducir al máximo estas pérdidas es necesario emplear sílice porosa de forma que el índice de refracción disminuya a 1,30 .

Los diferentes recubrimientos antirreflectantes comerciales se producen por pulverización catódica (3) y sol-gel (4-7), usando la via coloidal, con muy buenas propiedades ópticas pero pobres propiedades mecánicas por lo que sufren una fuerte degradación durante su uso a intemperie.

\section{PARTE EXPERIMENTAL.}

La solución precursora se prepara a partir de Tetraetilortosilicato (TEOS) hidrolizado en medio ácido con un contenido de Tritón entre 0 y $50 \mathrm{~g} / 1$.

Las películas se depositan por extracción a velocidad constante y son sinterizadas durante 10 minutos a $500^{\circ} \mathrm{C}$ con un soplante de aire caliente.

La transmitancia en el espectro solar se ha medido con un espec- trofotómetro Perkin-Elmer Lambda 9, dotado con una esfera integradora de sulfato de bario. La integración de la transmitancia se ha realizado con el espectro solar AM 1,5.

Las medidas de nanoindentación fueron realizadas con un Nanoindenter II's (Nano Instruments, Inc., Knoxville, TN). Cada una de los recubrimientos fue medido a temperatura ambiente empleando la técnica de nanoindentación continua $(8,9)$. Esta técnica permite obtener, de una manera continua y con una única indentación, la dureza y el módulo de elasticidad de la muestra a medir en función de la profundidad de contacto del indentador. Los resultados presentados en este trabajo se obtuvieron promediando los valores obtenidos de 10 indentaciones en ventanas de desplazamiento de $25 \mathrm{~nm}$.

\section{RESULTADOS Y DISCUSIÓN.}

Las soluciones precursoras de las capas antirreflectantes de sílice presentan una excelente estabilidad antes y después de la adición del Tritón usado para promover la porosidad. Se han realizado medidas semanales de la viscosidad a lo largo de cuatro meses sin observarse cambio alguno. Las películas obtenidas presentan una excelente homogeneidad, sin grietas ni defectos.

Las propiedades ópticas se ven fuertemente afectadas por el contenido de tritón en la solución precursora. En la gráfica 1 se puede apreciar como al aumentar el contenido de tritón, la transmitancia aumenta claramente. Para contenidos superiores a $50 \mathrm{~g} / 1$ se empiezan a producir problemas de homogeneidad y adherencia de las películas, sin producirse una mejora en las propiedades ópticas.

Para optimizar la transmitancia se empleó la solución precursora con $50 \mathrm{~g} / \mathrm{l}$ de Tritón y se realizaron recubrimientos a diferentes velocidades de extracción para obtener el espesor adecuado. En la gráfica 2 
se muestra el efecto del espesor de la capa AR sobre la transmitancia. Como se puede apreciar, al aumentar el espesor de la capa de sílice se produce un aumento de la altura del máximo y este se desplaza a longitudes de onda superiores. La máxima transmitancia integrada con el espectro solar AM 1.5 se obtiene a una velocidad de $16 \mathrm{~cm} / \mathrm{min}$, pasando de 0,917 para el vidrio sin recubrir a 0,970 con la capa AR optimizada.

El valor de transmitancia obtenido es muy elevado, siendo similar al de otros recubrimientos antirreflectantes presentados en la bibliografía y obtenidos mediante sol-gel, usando soluciones coloidales. La principal diferencia reside en que dichas películas presentan unas propiedades mecánicas muy pobres debido a la débil unión existente entre las partículas coloidales de sílice al sustrato y entre ellas mismas. Esto hace que los vidrios recubiertos no puedan ser limpiados sin dañar el recubrimiento y que su durabilidad ambiental sea muy baja.

Los recubrimientos antirreflectantes aquí presentados se obtienen a partir de soluciones poliméricas, en las que se han formado cadenas poliméricas tridimensionales con pequeñas micelas esféricas de Tritón. $\mathrm{Al}$ sinterizar las películas se produce la eliminación del aditivo, de forma que la capa de sílice obtenida presenta la estructura de una capa de sílice polimérica con los huecos dejados por el aditivo, de forma que las uniones entre las moléculas de sílice son muy fuertes.

Para evaluar el efecto de la adición del Tritón sobre las propiedades mecánicas se ha medido la dureza de las películas con y sin aditivo. En la gráfica 3 se muestra la evolución de la dureza de los recubrimientos de sílice densa y porosa en función de la profundidad de contacto del indentador. La dureza de los recubrimientos presenta unos valores constantes en la región cercana a la superficie de la muestra. Podemos considerar estos resultados como libres del efecto del substrato y, por lo tanto, propios del recubrimiento. Los valores de dureza obtenidos en esta región son de $0,66 \pm 0,03$ GPa para la sílice sin aditivo y de $0,49 \pm$ 0,05 GPa para la sílice porosa. Esta disminución observada, cercana al $25 \%$, es debida a la distinta estructura y menor densidad del recubrimiento poroso.

Considerando que la sílice fundida tiene una dureza cercana a 10 GPa, el valor obtenido para ambas capas de sílice es bajo, pero concuerda con los resultados mostrados en la bibliografía para películas obtenidas por sol-gel a partir de TEOS y catálisis ácida (10). La dureza de estas películas aumenta de forma exponencial con la temperatura de densificación, aumentando fuertemente a partir de $800^{\circ} \mathrm{C}$. Como es evidente, utilizando vidrio como sustrato, la temperatura máxima del tratamiento térmico no debe exceder los $500^{\circ} \mathrm{C}$, por lo que la dureza de las películas va a tener siempre valores discretos en comparación con la sílice fundida.

Con el fin de comparar la estabilidad mecánica de los recubrimientos preparados, con los comerciales, se realizaron medidas de adhesión con el ensayo de cinta adhesiva Schott (11) y utilizando papel de filtro como abrasivo. Todos los recubrimientos ensayados no presentaron degradación alguna al retirar de un tirón la cinta adhesiva de la superficie. Los recubrimientos comerciales se eliminan completamente en pocos segundos frotando con papel de filtro, mientras que el recubrimiento presentado en este trabajo no presenta ningún daño tras el proceso.

Con el fin de realizar un análisis más exhaustivo, tanto de las propiedades mecánicas como de la durabilidad ambiental, se van a estudiar los recubrimientos dentro de la Tarea 27 de la IEA.

\section{CONCLUSIONES.}

Los recubrimientos antirreflectantes presentados producen un aumento de la transmitancia del vidrio de 0,917 a 0,970. La dureza de los recubrimientos de sílice porosa se reduce un $25 \%$ frente a la misma capa obtenida sin el empleo del aditivo, pasando de 0,66 $\pm 0,03 \mathrm{GPa}$ a $0,49 \pm 0,05 \mathrm{Gpa}$. El estudio inicial de las propiedades mecánicas indica

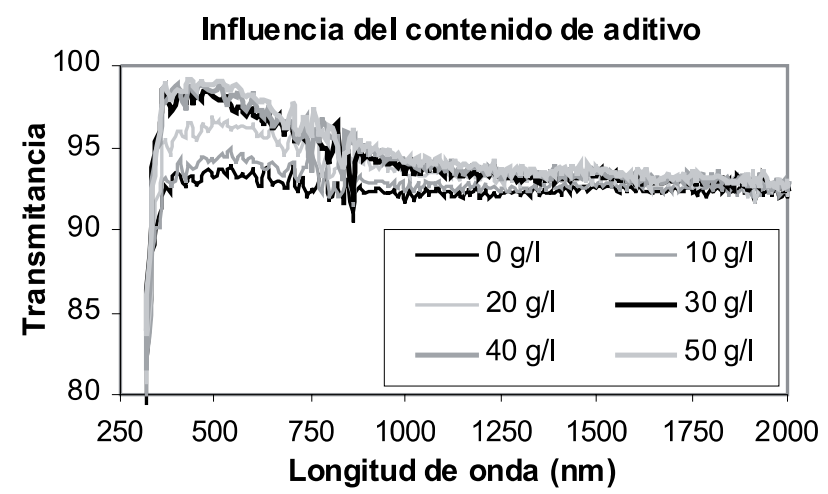

Figura 1. Influencia del contenido de tritón en la solución precursora sobre la transmitancia del vidrio recubierto.

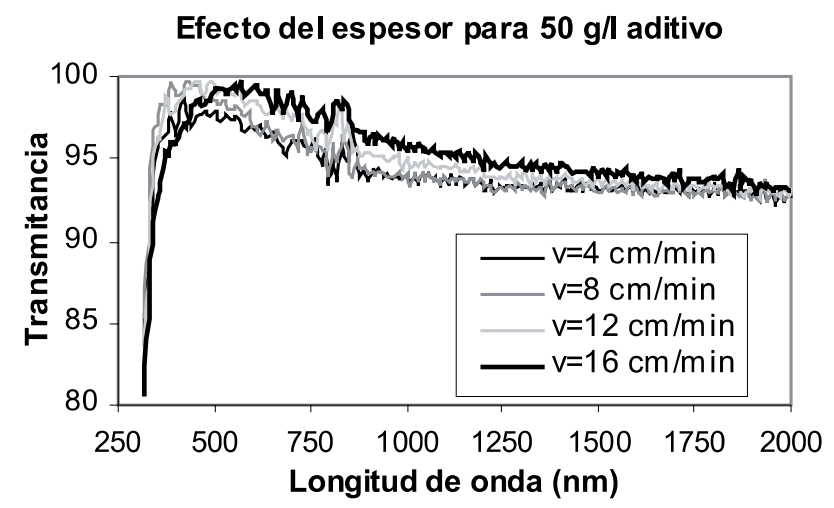

Figura 2. Influencia del espesor de la capa antirreflectante (velocidad de extracción del sustrato) sobre la transmitancia del vidrio recubierto para una solución con $50 \mathrm{~g} / 1$ de aditivo.

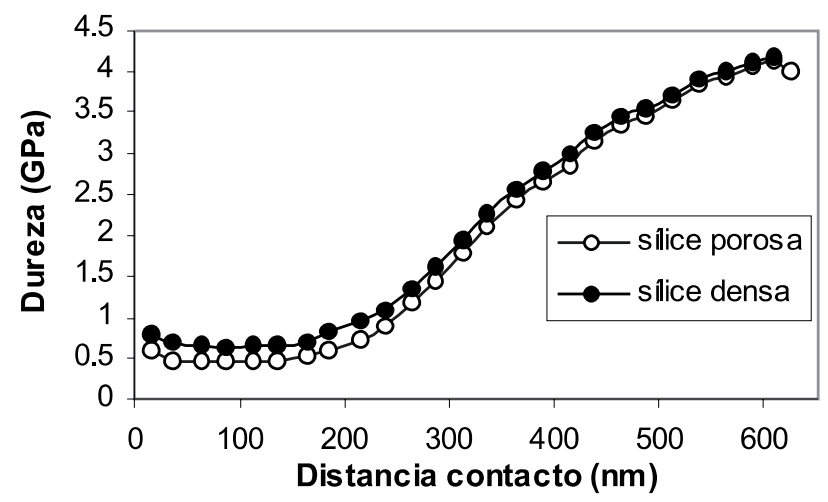

Figura 3. Variación de la dureza de la sílice densa (sin aditivo) y la sílice porosa (con aditivo) promediando los valores obtenidos de 10 indentaciones en ventanas de desplazamiento de $25 \mathrm{~nm}$.

que la resistencia mecánica de este recubrimiento es muy superior a la de los recubrimientos comerciales.

\section{BIBLIOGRAFÍA.}

1. K.W. Böer, “Advances in solar energy”, Plenum Press, New York, 1986. 2. R.B.Petit, C.J. Brinker, Solar Energy Materials 14 (1986) 269-287.

3. Solel Solar Systems LTD., Har-Hotzvim, P.O. Box 91450 Jerusalem, Israel.

4. M.T. Scholz, W.L. Kausch, D.R. Boston, J.M. Zoborowski, ES Patent No. 2153908T3, March 2001, Minnesota Mining and Manufacturing Co.

5. R. W. Lange, H.G. Sowman, S-K Kang, US Patent No. 4816333, March 1989, Minnesota Mining and Manufacturing Co.

6. B.E. Yoldas, US Patent No. 4271210, Jun 1981, Westinghouse Electric Corp.

7. H. Li, A.E. Wang, S.K. Das, US Patent No. 5580819, Dec. 1996, assigned to PPG Industries, Inc.

8. W.C. Oliver, G.M. Pharr, J. Mater. Res. 7(6) (1992) 1564.

9. J..B. Pethica and W.C. Oliver, Mater. Soc. Symp. Proc. 130, 13 (1989).

10. B.D. Fabes, W.C. Oliver, J. Non-Crys. Solids 121(1990)348-356.

11. D3359-97 Standard test methods for measuring adhesion by tape test. 